\title{
Constraining planet structure from stellar chemistry: the cases of CoRoT-7, Kepler-10, and Kepler-93 ${ }^{\star \star \star}$
}

\author{
N. C. Santos ${ }^{1,2}$, V. Adibekyan ${ }^{1}$, C. Mordasini ${ }^{3}$, W. Benz ${ }^{3}$, E. Delgado-Mena ${ }^{1}$, C. Dorn ${ }^{3}$, L. Buchhave ${ }^{4,5}$, P. Figueira ${ }^{1}$, \\ A. Mortier ${ }^{6}$, F. Pepe ${ }^{7}$, A. Santerne ${ }^{1}$, S. G. Sousa ${ }^{1}$, and S. Udry ${ }^{7}$ \\ ${ }^{1}$ Instituto de Astrofísica e Ciências do Espaço, Universidade do Porto, CAUP, Rua das Estrelas, 4150-762 Porto, Portugal \\ e-mail: nuno@astro.up.pt \\ 2 Departamento de Física e Astronomia, Faculdade de Ciências, Universidade do Porto, Rua Campo Alegre, 4169-007 Porto, Portugal \\ 3 Physikalisches Institut, University of Bern, Sidlerstrasse 5, 3012, Bern, Switzerland \\ ${ }^{4}$ Harvard-Smithsonian Center for Astrophysics, Cambridge, Massachusetts 02138, USA \\ 5 Centre for Star and Planet Formation, Natural History Museum of Denmark, Univ. of Copenhagen, 1350 Copenhagen, Denmark \\ 6 SUPA, School of Physics and Astronomy, University of St Andrews, St Andrews KY16 9SS, UK \\ 7 Observatoire de Genève, Université de Genève, 51 ch. des Maillettes, 1290 Sauverny, Switzerland \\ Received 29 June 2015 / Accepted 23 July 2015
}

\section{ABSTRACT}

\begin{abstract}
Aims. We explore the possibility that the stellar relative abundances of different species can be used to constrain the bulk abundances of known transiting rocky planets.

Methods. We use high resolution spectra to derive stellar parameters and chemical abundances for $\mathrm{Fe}, \mathrm{Si}, \mathrm{Mg}, \mathrm{O}$, and $\mathrm{C}$ in three stars hosting low mass, rocky planets: CoRoT-7, Kepler-10, and Kepler-93. These planets follow the same line along the mass-radius diagram, pointing toward a similar composition. The derived abundance ratios are compared with the solar values. With a simple stoichiometric model, we estimate the iron mass fraction in each planet, assuming stellar composition.

Results. We show that in all cases, the iron mass fraction inferred from the mass-radius relationship seems to be in good agreement with the iron abundance derived from the host star's photospheric composition.

Conclusions. The results suggest that stellar abundances can be used to add constraints on the composition of orbiting rocky planets.
\end{abstract}

Key words. planetary systems - planets and satellites: detection - stars: abundances - techniques: spectroscopic

\section{Introduction}

The study of chemical abundances in stars with planetary companions proved to be fundamental for our understanding of the formation of planetary systems as a whole (Ida \& Lin 2004; Mordasini et al. 2012). Important clues for the processes of planet formation and evolution have been brought by the discovery that the frequency of giant planets is a strong function of stellar metallicity (e.g. Santos et al. 2004; Fischer \& Valenti 2005), as well as by the fact that such a correlation does not seem to be present for stars hosting lower mass planets (Sousa et al. 2011; Buchhave et al. 2012) - see, however, discussions in Adibekyan et al. (2012a) and Wang \& Fischer (2015). Also, the stellar chemical composition seems to be related to the structure of the planets that were formed: the heavy element content of a giant planet seems to correlate with the host star metallicity (Guillot et al. 2006; Fortney et al. 2007). The whole planetary architecture (e.g., orbital periods and eccentricities) may be related to the presence of heavy elements in the stellar host (Beaugé \& Nesvorný 2013; Dawson \& Murray-Clay 2013; Adibekyan et al. 2013).

Specific relative abundances of chemical species may also be reflected in the bulk composition of the planets (e.g., Grasset et al. 2009). Different chemical abundances in the disk may

\footnotetext{
* Based on archival data obtained with the SOPHIE (1.93-m telescope OHP observatory), HARPS (3.6-m ESO, La SillaParanal Observatory), and HARPS-N (TNG telescope, La Palma) spectrographs.

$\star \star$ Appendices are available in electronic form at http://www . aanda.org
}

result in the formation of planets that have different composition and structure (Carter-Bond et al. 2012), something that may even change their habitability potential (Noack et al. 2014). Understanding whether the relative chemical abundances we measure in the photosphere of the host star are related to the relative bulk composition of its orbiting planet(s) may thus provide valuable clues for modeling planet structure once precise masses and radii are measured.

Recently, Dressing et al. (2015) has shown that five known rocky planets (Kepler-10b, Kepler-36b, Kepler-78b, Kepler-93b, and CoRoT-7b) with precise measurements of the mass (relative uncertainties less than 20\%) and radius all seem to fall along the same line in the mass-radius diagram: the area corresponding to an approximately Earth-like composition with a silicate mantle and an iron core of a similar mass fraction. Their best fit-model is comprised of $17 \%$ iron and $83 \%$ silicate mantle (MgSiO3), using a simple two-component interior structure model (Zeng \& Sasselov 2013). This is within $20 \%$ of the iron mass fractions estimates of Earth, Venus, and Mars with $36 \%, 30-36 \%$, and $23-25 \%$ derived, respectively (Reynolds \& Summers 1969), using a comparable model that assumes no iron oxides in the mantle. More detailed studies strongly suggest that relative abundances of $\mathrm{Fe}, \mathrm{Mg}$, and $\mathrm{Si}$ are similar among the Sun, Earth, Mars, Venus, as well as meteorites (e.g., Lodders 2003; Drake \& Righter 2002; Lodders \& Fegley 1998; Khan \& Connolly 2008; Sanloup et al. 1999). Meteorites are believed to be chemically similar to the building blocks of planets (Morgan \& Anders 1980), because both are condensates from the solar nebula that experienced the same fractionation processes. In the solar system the Sun's photospheric relative Fe-abundance can 
thus be used as a proxy for the iron mass fraction for three out of four terrestrial planets. This fraction is in turn a key quantity for the characterization of exoplanets: from measurement of mass and radius alone, the interior composition can only be weakly constrained owing to model degeneracies (Dorn et al. 2015).

It is thus interesting to see whether for the planets analyzed by Dressing et al. (2015), a compositional correlation between the host stars and their planets is possible, suggesting that it may not be restricted to the solar system case. This is predicted by planet formation models that include equilibrium condensation models corresponding to small distances to the host star ( $\sim 1 \mathrm{AU})$. As a consequence, these relative abundances do not vary significantly within a disk, whether spatially and temporally (e.g., Johnson et al. 2012; Thiabaud et al. 2014) ${ }^{1}$. If we find observational evidence that many exoplanets follow a mass-radius relation that agrees with the relative composition in refractories of their host stars, this would mean that we can use stellar composition as a proxy to reduce the degeneracy of possible interior models, more specifically, by coupling structure and composition of core and mantle through bulk abundance ratios (e.g. Dorn et al. 2015).

With the goal of testing these possibilities, we present a detailed study of the chemical abundances for three out of five of the planets discussed in Dressing et al. (2015): Kepler-10b, Kepler-93b, and CoRoT-7b. In Sects. 2 and 3 we present our data, the derivation of stellar parameters, and chemical abundances. We then discuss the results in Sect. 4.

\section{Observations and data}

Spectra for Kepler-10 and Kepler-93 were obtained from the SOPHIE archive (OHP 1.93-m telescope). The data was gathered as part of a program to derive masses and confirm the planetary nature of detected Kepler candidates (e.g., Santerne et al. 2012). The spectra cover the range between 3870 and $6940 \AA$. The spectra were obtained using the high resolution mode (HR; $R=75000$ ) for Kepler-10, and the high efficiency mode (HE; $R=40000$ ) for Kepler-93. In both cases Fiber B of the spectrograph was pointed toward a sky region. The spectra were reduced using the SOPHIE pipeline. The sky spectra were subtracted from the stellar spectrum using the information available in Fiber B after correcting for the efficiency difference between the two fibers. For Kepler-10 we combined five spectra obtained between 2011 July 7 and 2011 September 9 (other available spectra had very poor S/N). For Kepler-93 we combined two spectra obtained on the nights of 2014 March 13 and 2014 April 26. The $\mathrm{S} / \mathrm{N}$ of the final spectra is in the range of 80 and 60 as measured using continuum regions near $6500 \AA$, respectively.

For Kepler-10 we also gathered spectra obtained using the high resolution $(R=115000)$ HARPS-N spectrograph (Cosentino et al. 2012) - courtesy of the HARPS-N consortium. We combined a total of 110 high $\mathrm{S} / \mathrm{N}$ spectra $(S / N>50$ in order 50) obtained between 2012 May 25 and 2013 October 18. The final spectrum has a $\mathrm{S} / \mathrm{N}$ on the order of 600 . A comparison between the results obtained using the SOPHIE and HARPS-N spectra gives us an idea about the possible systematic errors between parameters and abundances derived using different spectra.

As we see below, we have already analyzed an existing HARPS spectrum for CoRoT-7. We point the reader to Mortier et al. (2013) for further details.

\footnotetext{
1 For a specific planet it is possible that an external process may have significantly altered its bulk composition like was the case for Mercury.
}

\section{Stellar parameters and abundances}

Stellar parameters for Kepler-10 (independently derived from the SOPHIE and HARPS-N spectra) and Kepler-93 were derived in LTE, using a grid of plane-parallel, ATLAS9 model atmospheres (Kurucz 1993) and the radiative transfer code MOOG (Sneden 1973). The methodology used is described in detail in Sousa et al. (2011). The full spectroscopic analysis is based on the equivalent widths (EWs) of $\sim 250 \mathrm{Fe}$ I and $40 \mathrm{Fe}$ II weak lines by imposing ionization and excitation equilibrium. Parameters for CoRoT-7 have already been derived by our team (using the same tools and methods) from a high resolution, high $\mathrm{S} / \mathrm{N}$ HARPS spectrum (Mortier et al. 2013).

For FGK dwarfs the effective temperatures obtained using this methodology were shown to be compatible with estimates from recent applications of the infrared flux method (see, e.g., Santos et al. 2013). Possible errors in the derived (always more uncertain) $\log g$ have little or no influence on the abundances of individual species as derived from atomic (non-ionized) lines.

The derived parameters for the three stars are listed in Table A.1, where we also list literature values for comparison. It is interesting that the parameters and abundances derived for Kepler-10 using the two spectra are similar despite the lower S/N and resolution of the SOPHIE data.

\subsection{Chemical abundances: $\mathrm{Si}, \mathrm{Mg}, \mathrm{C}$, and $\mathrm{O}$}

The abundances of magnesium $(\mathrm{Mg})$ and silicon ( $\mathrm{Si})$ were derived for the three stars following the same methodology as in our previous works (e.g., Adibekyan et al. 2012a, 2015). We used a LTE analysis relative to the Sun with the 2014 version of the code MOOG (Sneden 1973) and a grid of Kurucz ATLAS9 plane-parallel model atmospheres with no $\alpha$-enhancement. For the EW measurements, we used ARES2 (Sousa et al. 2015), for which the input parameters were the same as in Sousa et al. (2011). A visual inspection was done to confirm the reliability of the ARES2 measurements.

The abundances for each star and element were calculated from the average of the values provided by all measured lines (3 lines for $\mathrm{Mg}$ and 16 lines for $\mathrm{Si}$ ). A two-sigma clipping cut was performed to remove outliers. Errors in the abundances of the elements $[\mathrm{X} / \mathrm{H}]$ were evaluated adding quadratically the line-to-line scatter errors and errors induced by uncertainties in the model atmosphere parameters (see, e.g., Adibekyan et al. 2012a). The final abundances of $\mathrm{Mg}$ and $\mathrm{Si}$ are presented in Table A.1. For CoRoT-7, abundances for $\mathrm{Mg}$ and $\mathrm{Si}$ perfectly match the ones derived in Mortier et al. (2013) using an older version of MOOG.

Carbon $(\mathrm{C})$ and oxygen $(\mathrm{O})$ abundances were derived with the same tools as $\mathrm{Mg}$ and $\mathrm{Si}$ but measuring the EWs of the lines with the task splot in IRAF. Atomic data for the $\mathrm{O}$ lines and for the Ni line-blend affecting the oxygen forbidden line at $6300 \AA$ were taken from Bertran de Lis et al. (2015). The data for the atomic carbon lines was taken from the VALD3 database ${ }^{2}$.

The final abundances derived using each individual line are shown in Table A.2. The errors for the abundances are mainly due to the uncertainties in the position of the continuum. In the error estimate, we also considered the sensitivity of the $\mathrm{C}$ and $\mathrm{O}$ abundances due to the error in the atmospheric parameters, adding all terms quadratically as done for $\mathrm{Mg}$ and $\mathrm{Si}$. The error caused by the continuum placement was estimated by recalculating the abundance using different continuum positions as measured by hand. Unfortunately, the S/N of the SOPHIE spectra is

http://vald.astro.univie.ac.at/ vald3/php/vald.php 
N. C. Santos et al.: Constraining planet structure from stellar chemistry: the cases of CoRoT-7, Kepler-10, and Kepler-93

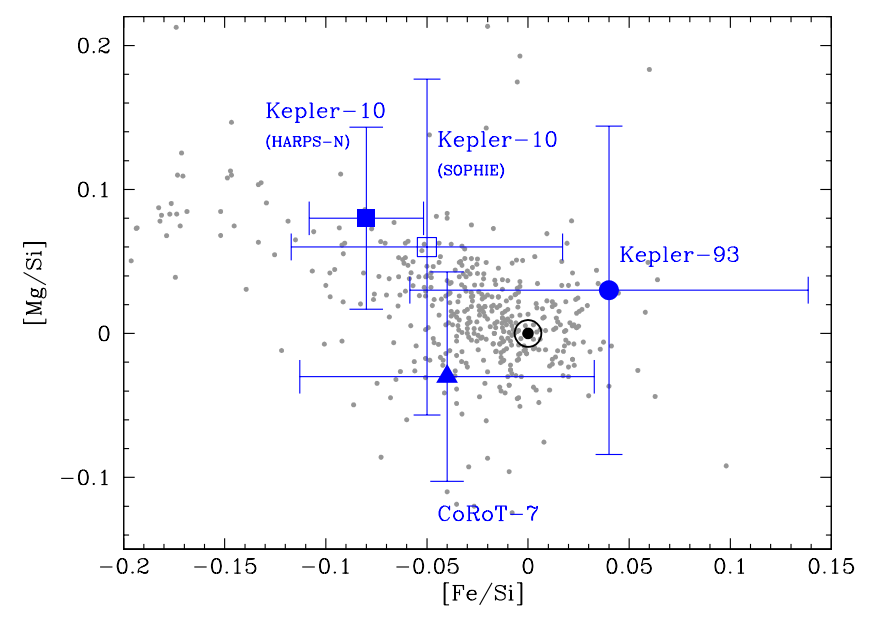

Fig. 1. $[\mathrm{Mg} / \mathrm{Si}]$ vs. $[\mathrm{Fe} / \mathrm{Si}]$ for the studied stars, together with the comparison dwarf (gray small dots) from Adibekyan et al. (2012b) with $T_{\text {eff }}=T_{\odot} \pm 300 \mathrm{~K}$. The position of the Sun is shown for comparison.

not high enough to allow us to measure the weak oxygen lines. Furthermore, the HARPS-N spectra for Kepler-10 shows telluric contamination in the region of the oxygen forbidden line. For CoRoT-7 the oxygen line at $6158 \AA$ is too weak. Therefore we could only derive $\mathrm{C} / \mathrm{O}$ ratios for Kepler-10 and CoRoT-7, based on a single oxygen indicator each.

For reference, the solar values are also shown Table A.2. While the carbon abundance in the Sun is well defined using the $\mathrm{CI}$ atomic lines, the oxygen abundance is more uncertain, and the different indicators (lines) provide different values (see discussion in Bertran de Lis et al. 2015). Therefore, in Table A. 2 we give the oxygen abundance values individually. The $[\mathrm{C} / \mathrm{O}]$ ratio is also computed using the corresponding solar abundance, depending on which oxygen line we could use.

\section{Discussion and conclusions}

In Fig. 1 we show the position of the stars in the $[\mathrm{Mg} / \mathrm{Si}]-[\mathrm{Fe} / \mathrm{Si}]$ plane. The plot shows that while Kepler-93 and CoRoT-7 do not seem to have significantly different abundance ratios than the Sun, the situation for Kepler-10 seems different. Both the abundances derived from the SOPHIE and HARPS-N spectra suggest that the star has a [Fe/Si] below solar $(-0.08 /-0.05$ from HARPS-N and SOPHIE data, respectively). The $[\mathrm{Mg} / \mathrm{Si}]$ ratio observed in Kepler-10 also seems to be above solar (0.08/0.06 from HARPS-N and SOPHIE data, respectively). In this sense it is interesting to see that both sets of spectra provide very similar results, even if the whole analysis was done in a fully independent way. It is worth noting that since the model changes (variation in stellar parameters) induce similar effects in the abundances of these refractory elements (they partially cancel out when computing the ratios [X1/X2]), our reported errors are likely conservative.

For Kepler-10 we find evidence for abundance ratios that are significantly different from solar, though the differences are not higher than $\sim 0.1$ dex. For CoRoT-7 and Kepler-93, on the other hand, the abundance ratios seem to be very similar to solar. Following the galactic chemical evolution trends (Bensby et al. 2005; Adibekyan et al. 2012b), we could expect that stars with $[\mathrm{Fe} / \mathrm{H}]$ below solar should on average present $[\mathrm{Si} / \mathrm{Fe}]$ abundances that are slightly above solar (i.e., [Fe/Si] ratios below solar), though perfectly allow the opposite trend given the observed dispersions.
Concerning $\mathrm{C}$ and $\mathrm{O}$, we first note that the carbon abundances for Kepler-10, as derived from different spectra (HARPS-N and SOPHIE), agree well within the errors. The $[\mathrm{C} / \mathrm{O}]$ ratio for this star seems to be subsolar. This is expected since oxygen, as a pure $\alpha$-element, grows more rapidly than carbon as a function of $[\mathrm{Fe} / \mathrm{H}]$, for $[\mathrm{Fe} / \mathrm{H}]<0$ (e.g., Bertran de Lis et al. 2015; Bensby et al. 2005). In any case, the observed [C/O] ratio values are not expected to have a significant impact on the bulk composition of the planet (Bond et al. 2010; Delgado Mena et al. 2010) when compared to a solar ratio.

On the other hand, for CoRoT-7 we could expect a $[\mathrm{C} / \mathrm{O}]$ ratio that is more similar to the solar value owing to its more similar metallicity. However, its elevated oxygen abundance yields a lower $[\mathrm{C} / \mathrm{O}]$ ratio. Although the determination of oxygen abundances can often be problematic for cooler stars such as CoRoT-7, in this case the quality of the spectrum allowed us to measure the EW of the forbidden line perfectly, and the spectrum does not seem to show telluric contamination in the region (which could produce an artificial enhanced oxygen abundance). We note that the high error of $[\mathrm{C} / \mathrm{O}]$ for this star precludes firm conclusions.

Whether the observed compositional differences are relevant when modeling the interior structure of the exoplanets is the next obvious question to answer. In particular, we want to know whether the interiors structure of the planets presented by Dressing et al. (2015) can be explained when using the specific stellar abundances as proxies for the planet bulk compositions. Given the large uncertainties on mass and radius, as well as on the abundances, we suppose that (1) this is likely the case and (2) the single model of an Earth-like iron-core mass fraction as used by Dressing et al. (2015) lies within the generally wide range of possible interior models. A detailed analysis of this including a full error analysis will be presented in future theoretical work. Here we only make first simple considerations regarding the relative expected abundances of the most important rock-forming elements and, in particular, of iron.

We only have abundances of $\mathrm{Fe}, \mathrm{Si}, \mathrm{Mg}, \mathrm{O}$, and $\mathrm{C}$. However, these rocky-forming elements, together with $\mathrm{H}$ and $\mathrm{He}$, are the most relevant ones for controlling the species expected from equilibrium condensation models, (Lodders 2003; Seager et al. 2007), namely $\mathrm{H}_{2}, \mathrm{He}, \mathrm{CH}_{4}, \mathrm{H}_{2} \mathrm{O}, \mathrm{Fe}, \mathrm{MgSiO}_{3}$, and $\mathrm{Mg}_{2} \mathrm{SiO}_{4}$ (all stars have $N_{\mathrm{Mg}}>N_{\mathrm{Si}}$ ). A simplified model for the expected mass fractions of different compounds using these species is thus a reasonable approach. In this chemical network, the molecular abundances and therefore the mass fraction can be found from the atomic abundances with simple stoichometry:

$N_{\mathrm{O}}=N_{\mathrm{H}_{2} \mathrm{O}}+3 N_{\mathrm{MgSiO}_{3}}+4 N_{\mathrm{Mg}_{2} \mathrm{SiO}_{4}}$
$N_{\mathrm{Mg}}=N_{\mathrm{MgSiO}_{3}}+2 N_{\mathrm{Mg}_{2} \mathrm{SiO}_{4}}$
$N_{\mathrm{Si}}=N_{\mathrm{MgSiO}_{3}}+N_{\mathrm{Mg}_{2} \mathrm{SiO}_{4}}$.

Similar equations hold for the other species. This way we calculated the expected mass fractions, in particular $f_{\text {iron}}$, for the three stars analyzed in this paper. Table 1 shows the result in terms of mass fractions $m_{\mathrm{X}}$ of the five heavy element species (in percentage points), the total mass fraction of heavy elements $Z$, and finally the iron mass fraction among the refractory elements, which is the key result. In the table we also showed, for comparison, the results with our basic chemistry model for the Sun using the solar composition of Asplund et al. (2009). The table also contains the condensate abundances derived by Lodders (2003, her Table 11, photospheric values) with a full condensation model that includes a much higher number of species. Kepler-93 (K-93) has no oxygen measurement. We assumed a 
Table 1. Mass fractions of heavy element, total fraction of heavy elements, and iron mass fraction among refractory species (values in \%).

\begin{tabular}{lccccc}
\hline \hline Quantity & C-7 & K-93 & K-10 & Sun & Sun $^{f}$ \\
\hline $\mathrm{H}_{2} \mathrm{O}^{a}$ & $0.75 \pm 0.31$ & $0.54 \pm 0.22$ & $0.98 \pm 0.20$ & 0.50 & 0.51 \\
$\mathrm{CH}^{a}$ & $0.32 \pm 0.05$ & $0.35 \pm 0.13$ & $0.36 \pm 0.04$ & 0.37 & 0.29 \\
$\mathrm{Fe}^{a}$ & $0.14 \pm 0.01$ & $0.09 \pm 0.01$ & $0.10 \pm 0.00$ & 0.13 & $0.17^{d}$ \\
$\mathrm{MgSiO}_{3}{ }^{a}$ & $0.25 \pm 0.08$ & $0.10 \pm 0.06$ & $0.11 \pm 0.05$ & 0.19 & $0.27^{e}$ \\
$\mathrm{Mg}_{2} \mathrm{SiO}_{4}{ }^{a}$ & $0.05 \pm 0.06$ & $0.08 \pm 0.06$ & $0.14 \pm 0.06$ & 0.08 & \\
\hline $\mathrm{Z}^{b}$ & $1.50 \pm 0.31$ & $1.17 \pm 0.25$ & $1.69 \pm 0.21$ & 1.26 & 1.32 \\
\hline$f_{\text {iron }}{ }^{c}$ & $31.6 \pm 2.6$ & $34.7 \pm 3.7$ & $27.5 \pm 1.7$ & 33.2 & 38.0 \\
\hline
\end{tabular}

Notes. ${ }^{(a)}$ The $m_{\mathrm{H} 2}$ and $m_{\mathrm{He}}$ are between $74.7-75.1 \%$ and $23.6-23.7 \%$, respectively. (b) Summed mass percent of all heavy elements. (c) $m_{\mathrm{Fe}} /\left(m_{\mathrm{Fe}}+m_{\mathrm{MgSiO} 3}+m_{\mathrm{Mg} 2 \mathrm{SiO} 4}\right)$. ${ }^{(d)}$ Includes all metal species and FeS. ${ }^{(e)}$ Includes all silicates and oxides. ${ }^{(f)}$ Lodders (2003).

solar oxygen abundance for this star. This is a good assumption given the expected oxygen-to-iron ratio for stars with such metallicity (e.g., Bertran de Lis et al. 2015). Error bars were derived as explained in Appendix B.

The table shows that owing to the approximately scaled solar composition of CoRoT-7 (C-7) and Kepler-93 (under the assumption of solar $N_{\mathrm{O}}$ ), their $f_{\text {iron }}$ is very similar to the one of the Sun (32-35\%). Only for Kepler-10 (K-10), which is more depleted in $\mathrm{Fe}$ than in $\mathrm{Mg}$ and $\mathrm{Si}$ relative to the $\mathrm{Sun}$, do we see a slightly lower $f_{\text {iron }}=27.5 \%$ (and higher $\mathrm{H}_{2} \mathrm{O}$ ), as expected. Curiously, both Kepler-10b and Kepler-10c seem to be "oversized" in the mass-radius diagram of Dressing et al. (2015) when compared to the Earth composition model. In any case, we find that for the three planets analyzed by Dressing et al. (2015), the iron mass fraction inferred from the mass-radius relationship $(\sim 17 \%)$ is on a $\sim 10-20 \%$ level, in agreement with the iron abundance derived here from the host star photospheric composition, and in even better agreement with the mass fraction ( 29-32\%) expected from more detailed models of solar system planets (for the Earth see, e.g., McDounough \& Sun 1995). If confirmed, this interesting result doubles the number of rocky planets (including the solar system's) where the mass of the iron core can be estimated if the stellar composition is known, and brings it to six out of seven rocky planets where such an inference can be made (the exception is Mercury). As discussed in the introduction, this is important for reducing the compositional degeneracy of planet interiors (Dorn et al. 2015) and for constraining impact events.

A much more detailed analysis that combines host star abundances, mass and radius measurements, and internal structure calculations should be done in future work, taking all the associated measurement and model uncertainties into account. If the agreement of the planetary core mass (derived from the $M-R$ relationship) and the stellar photospheric abundances found here is confirmed and extended to additional planets, this would allow a better characterization of exoplanet interiors, even if a significantly different composition (as for Mercury) cannot be excluded a priori for an individual planet. This shows that a detailed chemical analysis of exoplanet hosts is important not only for the architecture of a planetary system, but also for the interior structure, composition, and potential habitability of individual planets. The detailed characterization of planets discovered and characterized by future space missions such as TESS, CHEOPS, and PLATO-2.0 will certainly gain from this analysis.

Acknowledgements. We would like to thank the anonymous referee for the useful comments and suggestions. This work was supported by Fundação para a Ciência e a Tecnologia (FCT) through the research grant UID/FIS/04434/2013.
P.F., N.C.S., and S.G.S. also acknowledge support from FCT through Investigador FCT contracts of reference IF/01037/2013, IF/00169/2012, and IF/00028/2014, and POPH/FSE (EC) by FEDER funding through the program "Programa Operacional de Factores de Competitividade - COMPETE". P.F. acknowledges support from FCT in the form of project reference IF/01037/2013CP1191/CT0001. A.S. is supported by the EU under a Marie Curie Intra-European Fellowship for Career Development with reference FP7-PEOPLE-2013-IEF, number 627202. E.D.M. and V.A. acknowledge the support from FCT in form of the grants SFRH/BPD/76606/2011 and SFRH/BPD/70574/2010. This work results within the collaboration of the COST Action TD 1308. C.M. acknowledges the support from the Swiss National Science Foundation under grant BSSGI0_155816. A.M. received funding from the European Union Seventh Framework Program (FP7/2007-2013) under grant agreement number 313014 (ETAEARTH).

\section{References}

Adibekyan, V. Z., Delgado Mena, E., Sousa, S. G., et al. 2012a, A\&A, 547, A36 Adibekyan, V. Z., Sousa, S. G., Santos, N. C., et al. 2012b, A\&A, 545, A32 Adibekyan, V. Z., Figueira, P., Santos, N. C., et al. 2013, A\&A, 560, A51 Adibekyan, V. Z., Benamati, L., Santos, N. C., et al. 2015, MNRAS, 450, 1900 Asplund, M., Grevesse, N., Sauval, A. J., \& Scott, P. 2009, ARA\&A, 47, 481 Ballard, S., Chaplin, W. J., Charbonneau, D., et al. 2014, ApJ, 790, 12 Batalha, N. M., Borucki, W. J., Bryson, S. T., et al. 2011, ApJ, 729, 27 Beaugé, C., \& Nesvorný, D. 2013, ApJ, 763, 12

Bensby, T., Feltzing, S., Lundström, I., \& Ilyin, I. 2005, A\&A, 433, 185 Bertran de Lis, S., Delgado Mena, E., Adibekyan, V. Z., Santos, N. C., \& Sousa, S. G. 2015, A\&A, 576, A89

Bond, J. C., O'Brien, D. P., \& Lauretta, D. S. 2010, ApJ, 715, 1050

Bruntt, H., Deleuil, M., Fridlund, M., et al. 2010, A\&A, 519, A51

Buchhave, L. A., Latham, D. W., Johansen, A., et al. 2012, Nature, 486, 375

Carter-Bond, J. C., O’Brien, D. P., \& Raymond, S. N. 2012, ApJ, 760, 44

Cosentino, R., Lovis, C., Pepe, F., et al. 2012, in SPIE Conf. Ser. 8446, 1

Dawson, R. I., \& Murray-Clay, R. A. 2013, ApJ, 767, L24

Delgado Mena, E., Israelian, G., González Hernández, J. I., et al. 2010, ApJ, 725, 2349

Dorn, C., Khan, A., Heng, K., et al. 2015, A\&A, 577, A83

Drake, M. J., \& Righter, K. 2002, Nature, 416, 39

Dressing, C. D., Charbonneau, D., Dumusque, X., et al. 2015, ApJ, 800, 135

Dumusque, X., Bonomo, A. S., Haywood, R. D., et al. 2014, ApJ, 789, 154

Fischer, D. A., \& Valenti, J. 2005, ApJ, 622, 1102

Fortney, J. J., Marley, M. S., \& Barnes, J. W. 2007, ApJ, 659, 1661

Grasset, O., Schneider, J., \& Sotin, C. 2009, ApJ, 693, 722

Guillot, T., Santos, N. C., Pont, F., et al. 2006, A\&A, 453, L21

Ida, S., \& Lin, D. N. C. 2004, ApJ, 616, 567

Johnson, T. V., Mousis, O., Lunine, J. I., \& Madhusudhan, N. 2012, ApJ, 757, 192

Khan, A., \& Connolly, J. A. D. 2008, J. Geophys. Res., 113, E07003

Kurucz, R. 1993, CD-ROM No. 13 (Cambridge, Mass.: Smithsonian Astrophysical Observatory)

Léger, A., Rouan, D., Schneider, J., et al. 2009, A\&A, 506, 287

Lodders, K. 2003, ApJ, 591, 1220

Lodders, K., \& Fegley, B. 1998, The planetary scientist's companion (New York: OUP)

McDounough, W. F., \& Sun, S.-s. 1995, Chemical Geology, 120, 223

Mordasini, C., Alibert, Y., Benz, W., Klahr, H., \& Henning, T. 2012, A\&A, 541, A97

Morgan, J. W., \& Anders, E. 1980, PNAS, 77, 6973

Mortier, A., Santos, N. C., Sousa, S. G., et al. 2013, A\&A, 558, A106

Noack, L., Godolt, M., von Paris, P., et al. 2014, Planet. Space Sci., 98, 14

Reynolds, R. T., \& Summers, A. L. 1969, J. Geophys. Res., 74, 2494

Sanloup, C., Jambon, A., \& Gillet, P. 1999, Physics of the Earth and Planetary Interiors, 112, 43

Santerne, A., Díaz, R. F., Moutou, C., et al. 2012, A\&A, 545, A76

Santos, N. C., Israelian, G., \& Mayor, M. 2004, A\&A, 415, 1153

Santos, N. C., Sousa, S. G., Mortier, A., et al. 2013, A\&A, 556, A150

Seager, S., Kuchner, M., Hier-Majumder, C. A., \& Militzer, B. 2007, ApJ, 669, 1279

Sneden, C. 1973, Ph.D. Thesis, Austin, Univ. of Texas

Sousa, S. G., Santos, N. C., Israelian, G., Mayor, M., \& Udry, S. 2011, A\&A 533, A141

Sousa, S. G., Santos, N. C., Adibekyan, V., Delgado-Mena, E., \& Israelian, G. 2015, A\&A, 577, A67

Thiabaud, A., Marboeuf, U., Alibert, Y., et al. 2014, A\&A, 562, A27

Wang, J., \& Fischer, D. A. 2015, AJ, 149, 14

Zeng, L., \& Sasselov, D. 2013, PASP, 125, 227

\section{Page 5 is available in the electronic edition of the journal at http://www . aanda.org}


N. C. Santos et al.: Constraining planet structure from stellar chemistry: the cases of CoRoT-7, Kepler-10, and Kepler-93

\section{Appendix A: Stellar parameters and chemical abundances}

Table A.1. Stellar atmospheric parameters and iron, magnesium, and silicon abundances for our targets.

\begin{tabular}{|c|c|c|c|c|c|c|c|c|}
\hline Star & $\begin{array}{l}T_{\text {eff }} \\
(\mathrm{K})\end{array}$ & $\begin{array}{l}\log g \\
(\operatorname{dex})\end{array}$ & $\begin{array}{c}\xi \\
\left(\mathrm{km} \mathrm{s}^{-1}\right)\end{array}$ & $\begin{array}{c}{[\mathrm{Fe} / \mathrm{H}]} \\
(\mathrm{dex})\end{array}$ & $\begin{array}{c}{[\mathrm{Mg} / \mathrm{H}]} \\
(\mathrm{dex})\end{array}$ & $\begin{array}{c}{[\mathrm{Si} / \mathrm{H}]} \\
(\mathrm{dex})\end{array}$ & Source & Instrument \\
\hline Kepler-10 & $5671 \pm 17$ & $4.34 \pm 0.01$ & $0.92 \pm 0.02$ & $-0.14 \pm 0.02$ & $+0.02 \pm 0.06$ & $-0.06 \pm 0.02$ & This paper & HARPS-N \\
\hline Kepler-10 & $5614 \pm 39$ & $4.27 \pm 0.04$ & $0.82 \pm 0.05$ & $-0.14 \pm 0.03$ & $-0.03 \pm 0.10$ & $-0.09 \pm 0.06$ & This paper & SOPHIE \\
\hline Kepler-93 & $5689 \pm 48$ & $4.56 \pm 0.06$ & $1.02 \pm 0.07$ & $-0.15 \pm 0.04$ & $-0.16 \pm 0.07$ & $-0.19 \pm 0.09$ & This paper & SOPHIE \\
\hline CoRoT-7 & $5288 \pm 27$ & $4.40 \pm 0.07$ & $0.90 \pm 0.05$ & $+0.02 \pm 0.02$ & $+0.03 \pm 0.03$ & $+0.07 \pm 0.08$ & Mortier et al. (2013) & HARPS \\
\hline \multicolumn{9}{|c|}{ Literature values: } \\
\hline \multirow[t]{2}{*}{ Kepler-10 } & $5627 \pm 44$ & $4.34 \pm 0.01$ & & $-0.15 \pm 0.04$ & & & Batalha et al. (2011) & \\
\hline & $5721 \pm 26$ & 4.34 & & $-0.14 \pm 0.02$ & & & Dumusque et al. (2014) & \\
\hline Kepler-93 & $5669 \pm 75$ & $4.47 \pm 0.01$ & & $-0.18 \pm 0.10$ & & & Ballard et al. (2014) & \\
\hline \multirow[t]{2}{*}{ CoRoT-7 } & $5250 \pm 60$ & $4.47 \pm 0.05$ & & $+0.12 \pm 0.06$ & & & Bruntt et al. (2010) & \\
\hline & $5275 \pm 75$ & $4.50 \pm 0.10$ & & $+0.03 \pm 0.06$ & & & Léger et al. (2009) & \\
\hline
\end{tabular}

Table A.2. Carbon and oxygen abundances (in dex) for our stars and the Sun, using the Kurucz Solar Atlas (Kurucz et al. 1984).

\begin{tabular}{lccccccccc}
\hline \hline Star & $\log \mathrm{C}_{5052 \AA}$ & $\log \mathrm{C}_{5380 \AA}$ & $\log \mathrm{O}_{6158 \AA}$ & $\log \mathrm{O}_{6300 \AA}$ & {$[\mathrm{C} / \mathrm{H}]$} & {$[\mathrm{O} / \mathrm{H}]$} & {$[\mathrm{C} / \mathrm{O}]$} & $\mathrm{C} / \mathrm{O}^{e}$ & $\mathrm{Mg} / \mathrm{Si}^{e}$ \\
\hline Kepler-10 $^{b}$ & 8.48 & 8.47 & 8.92 & - & $-0.01 \pm 0.05$ & $0.21 \pm 0.08$ & $-0.22 \pm 0.10$ & 0.36 & 1.48 \\
Kepler-10 $^{a}$ & 8.52 & 8.44 & - & - & $0.00 \pm 0.08$ & - & - & - & 1.41 \\
Kepler-93 $^{a}$ & 8.35 & 8.59 & - & - & $-0.02 \pm 0.16$ & - & - & - & 1.32 \\
CoRoT-7 $^{c}$ & 8.38 & 8.48 & - & 8.83 & $-0.06 \pm 0.07$ & $0.18 \pm 0.15$ & $-0.24 \pm 0.17$ & 0.40 & 1.12 \\
Sun & 8.48 & 8.49 & 8.71 & 8.65 & 0.00 & 0.00 & 0.00 & $0.64^{d}$ & 1.23 \\
\hline
\end{tabular}

Notes. ${ }^{(a)}$ SOPHIE. ${ }^{(b)}$ HARPS-N. ${ }^{(c)}$ HARPS. ${ }^{(d)} \mathrm{C} / \mathrm{O}$ ratios derived using the average values for the oxygen and carbon abundances given by different lines. ${ }^{(e)} A / B=N_{A} / N_{B}=10^{\log \varepsilon(A)} / 10^{\log \varepsilon(B)}$, where $\log \varepsilon(A)$ and $\log \varepsilon(B)$ are the absolute abundances.

\section{Appendix B: Errors}

The errors in the mass fractions were calculated using a Monte Carlo approach. We randomly drew $10^{5}$ values of the derived stellar abundances abundances following a Gaussian distribution centered on the derived abundance and with a sigma corresponding to the derived errors as listed in Tables A.1 and A.2. For each drawn set of $[\mathrm{Si} / \mathrm{H}],[\mathrm{Mg} / \mathrm{H}],[\mathrm{O} / \mathrm{H}]$, and $[\mathrm{C} / \mathrm{H}]$, we then computed the expected fractions. The distribution of the resulting values allowed us to derive the one-sigma error, as listed in Table 1.

We note that this procedure was done in a self consistent way. All the mass fractions were computed simultaneously for each set of input abundances. This is important because the resulting mass fraction values are correlated between themselves.

For Kepler-93, since the oxygen abundance was assumed to be solar (no $\mathrm{O}$ abundance could be derived from our data), we considered that the $[\mathrm{O} / \mathrm{H}]$ has an uncertainty of 0.15 dex. 